\title{
How Twitter conversations using hashtags \#regionalanesthesia and \#regionalanaesthesia have changed in the COVID-19 era
}

\author{
Eric S Schwenk (D) ,' Kellie M Jaremko, ${ }^{2}$ Rajnish K Gupta (D) , ${ }^{3}$ Nabil M Elkassabany, ${ }^{4}$ \\ Amit Pawa, ${ }^{5}$ Alex Kou, ${ }^{6,7}$ Edward R Mariano (D) ${ }^{6,7}$
}

'Department of Anesthesiology, Sidney Kimmel Medical College at Thomas Jefferson University, Philadelphia, Pennsylvania, USA ${ }^{2}$ Department of Anesthesia, Critical Care and Pain Medicine, Massachusetts General Hospital, Boston, Massachusetts, USA ${ }^{3}$ Department of Anesthesiology, Vanderbilt University Medical Center, Nashville, Tennessee, USA

${ }^{4}$ Department of Anesthesiology and Critical Care, University of Pennsylvania, Philadelphia, Pennsylvania, USA

${ }^{5}$ Department of Anaesthesia, Guy's and St Thomas' National Health Service Foundation Trust, London, UK

${ }^{6}$ Department of Anesthesiology, Perioperative and Pain Medicine, Stanford University School of Medicine, Stanford, California, USA

${ }^{7}$ Anesthesiology and Perioperative Care Service, VA Palo Alto Health Care System, Palo Alto, California, USA

Correspondence to Dr Edward R Mariano Anesthesiology and Perioperative Care Service, VA Palo Alto Health Care System, Palo Alto CA 94304, California, USA; emariano@stanford.edu

Received 26 May 2020 Revised 17 June 2020 Accepted 19 June 2020 Published Online First 2 July 2020

Check for updates

(C) American Society of Regional Anesthesia \& Pain Medicine 2020. No commercial re-use. See rights and permissions. Published by BMJ.

To cite: Schwenk ES,

Jaremko KM, Gupta RK,

et al. Reg Anesth Pain Med

2020:45:765-766

\section{INTRODUCTION}

Within the regional anesthesiology and acute pain medicine (RAAPM) Twitter community, the two common hashtags are \#regionalanesthesia and \# regionalanaesthesia. ${ }^{1}$ Hashtags (words/phrases following a "\#" symbol) identify themed tweets.

Before COVID-19, a common RAAPM topic was opioids, and many fear the opioid epidemic will worsen post-pandemic. ${ }^{2}$ We tested the hypothesis that the proportion of \#regionalanesthesia and \# regionalanaesthesia tweets related to opioids has decreased since COVID-19.

\section{METHODS}

This project was deemed exempt by the institutional review board.

\section{Study sample}

English language tweets including \#regionalanesthesia or \#regionalanaesthesia were prospectively collected using TAGS V.6.1.9.1. ${ }^{3}$ The first tweet in either hashtag archive to reference COVID-19 was on March 18, 2020. We therefore set our convenience sample from February 1 through April 30, 2020, to compare tweets 6 weeks before (pre) and after (post) this seminal tweet. We included original tweets, replies, and retweets. We excluded duplicates and tweets lacking either hashtag.

\section{Primary outcome}

The primary outcome was the proportion of tweets during each time interval referencing opioids. Microsoft Excel (Redmond, Washington, USA) was used to search for opioid terminology and opioid names.

\section{Secondary outcomes}

Microsoft Excel was used to search broadly for terms related to COVID-19. Users were categorized manually by ERM and KMJ using Symplur Healthcare Stakeholder Definitions. ${ }^{4}$ Tweets were assigned to one of four published categories: scientific, logistical, social, or other. ${ }^{5}$ Inter-rater reliability for tweet categorization was determined using Cohen's kappa statistic. The top 10 influencers were determined by tweets and impressions. ${ }^{5}$

\section{Statistical analysis}

Statistical analysis was performed using NCSS Statistical Software (NCSS, LLC, Kaysville, Utah,
USA) and IBM SPSS Statistics V.23 (IBM Corp., Armonk, New York, USA). The $\chi^{2}$ test with Yates correction was used for all comparisons of categorical data. For the primary outcome, a two-sided $\mathrm{p}<0.05$ was considered statistically significant. All other analyzes were considered exploratory and not adjusted for multiple comparisons.

\section{RESULTS}

From 1603 individual tweets with \#regionalanesthesia or \#regionalanaesthesia, 1268 tweets comprised the final sample after de-duplication: 780 pre (210 original, 561 retweets, and 9 replies) and 488 post (184 original, 287 retweets, and 17 replies). Retweets decreased from $71.9 \%$ pre to $58.8 \%$ post $(\mathrm{p}<0.001)$; original tweets increased from $26.9 \%$ pre to $37.7 \%$ in the post interval $(\mathrm{p}<0.001)$.

\section{Primary outcome}

Opioid tweets decreased from $2.7 \%$ (21/780) pre to $0.4 \%(2 / 488)$ post $(p=0.006)$.

\section{Secondary outcomes}

COVID-19 tweets increased from $0 \%$ pre to $26.6 \%(130 / 488)$ post $(\mathrm{p}<0.001)$. Doctors had the largest tweet decrease ( -242 tweets), from 59.7\% $(466 / 780)$ pre to $45.9 \%(224 / 488)$ post $(\mathrm{p}<0.001)$.

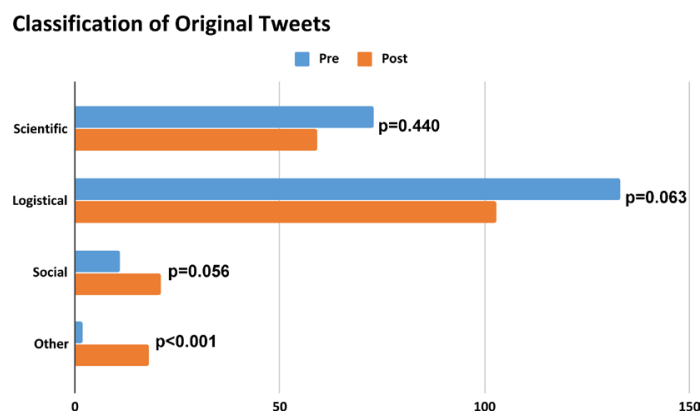

Figure 1 Classification of original tweets using categories from Schwenk et al $\left.\right|^{5}$ : scientific (contained education, shared conference-related content, or other form of medical education; logistical (broadcasted information such as an announcement about an upcoming conference or job opening); social (general thoughts, banter or conversation, and replies); or other (any tweet that did not obviously fall into one of the other three categories, including advertising tweets). $\mathrm{P}$ values are derived from the $\chi^{2}$ test with Yates correction. 
Research report

Table 1 Top 10 influencers of \#regionalanesthesia or \#regionalanaesthesia by number of tweets and number of impressions

\begin{tabular}{|c|c|c|c|c|c|c|c|}
\hline \multicolumn{4}{|l|}{ By tweets } & \multicolumn{4}{|l|}{ By impressions } \\
\hline Pre & & Post & & Pre & & Post & \\
\hline amit_pawa & 85 & cdrrogers & 55 & amit_pawa & 598400 & MDJobSite & 361725 \\
\hline crnajobsite & 47 & crnajobsite & 49 & MDJobSite & 340830 & ASRA_Society & 194264 \\
\hline RegionalAnaesUK & 45 & MDJobSite & 35 & EMARIANOMD & 279422 & EMARIANOMD & 134860 \\
\hline MDJobSite & 35 & medovate & 18 & RegionalAnaesUK & 244710 & amit_pawa & 106890 \\
\hline EMARIANOMD & 22 & ASRA_Society & 14 & ASRA_Society & 92358 & RegionalAnaesUK & 64064 \\
\hline LSORA_UK & 19 & amit_pawa & 14 & Anaes_Journal & 88332 & anesthesianews & 57894 \\
\hline Steve_Coppens & 18 & dr_rajgupta & 12 & ESRA_Society & 82264 & cdrrogers & 48510 \\
\hline claralexlobo & 13 & RegionalAnaesUK & 11 & LSORA_UK & 82137 & dr_rajgupta & 43644 \\
\hline canestezi & 12 & EMARIANOMD & 10 & Wilkinsonjonny & 40344 & crnajobsite & 42140 \\
\hline Abelgavino & 11 & KalagaraHari & 9 & TomVargheseJr & 38128 & BJAJournals & 25386 \\
\hline
\end{tabular}

Individual other health showed the largest tweet increase (+48 tweets), from $1.3 \%(10 / 780)$ pre to $11.9 \%$ (58/488) post $(\mathrm{p}<0.001)$. Cohen's kappa statistic was 0.796 ("substantial" agreement between reviewers) ${ }^{6}$ for the categorization of 420 original tweets. From pre to post, the proportion of "other" tweets increased (figure 1). All 20 tweets in the "other" category were medical device advertisements.

\section{Comparison of influencers}

The top 10 influencers of \# regionalanesthesia or \#regionalanaesthesia in the pre and post intervals are shown in table 1.

\section{DISCUSSION}

This study reveals a focus shift within the RAAPM Twitter community since COVID-19 arrived with fewer mentions of the opioid epidemic and more industry advertisements using regional anesthesia hashtags. However, an overall decrease in activity among the top 10 influencers also occurred, which may relate to increased clinical demands on anesthesiologists during COVID-19 or an appropriate change in topical priorities to personal protective equipment and critical care skills. Given the ongoing overlap of the COVID-19 pandemic and opioid epidemic, we encourage physicians in the RAAPM Twitter community to continue to use these hashtags to help disseminate information on opioids and nerve blocks as elective surgeries and normal clinical activities resume.

Twitter Eric S Schwenk @ESchwenkMD, Kellie M Jaremko @Neuro_Kellie, Rajnish K Gupta @dr_rajgupta, Amit Pawa @amit_pawa and Edward R Mariano @ EMARIANOMD

Acknowledgements This material is the result of work supported with resources and the use of facilities at the Veterans Affairs (VA) Palo Alto Health Care System (Palo Alto, California, USA). The contents do not represent the views of the Department of Veterans Affairs or the US Government.

Contributors ESS helped design the study, collected and analyzed data, drafted and revised the initial manuscript, reviewed and approved the submitted manuscript. $\mathrm{KJ}$ helped design the study, collected and analyzed data, drafted and revised the initial manuscript, reviewed and approved the submitted manuscript. RKG helped design the study, reviewed and approved the submitted manuscript. NME helped design the study, reviewed and approved the submitted manuscript. AP helped design the study, reviewed and approved the submitted manuscript. AK collected and analyzed data, reviewed and approved the submitted manuscript. ERM helped design the study, collected and analyzed data, drafted and revised the initial manuscript, reviewed and approved the submitted manuscript.

Funding The authors have not declared a specific grant for this research from any funding agency in the public, commercial or not-for-profit sectors.

Competing interests AP has received honoraria from GE Healthcare and consults for B. Braun Medical (Sheffield, South Yorkshire, UK). RKG consults for MedCreds (San Francisco, California, USA). NME consults for Foundry Therapeutics (Menlo Park, California, USA)

Patient consent for publication Not required.

Provenance and peer review Not commissioned; externally peer reviewed.

This article is made freely available for use in accordance with BMJ's website terms and conditions for the duration of the covid-19 pandemic or until otherwise determined by BMJ. You may use, download and print the article for any lawful, non-commercial purpose (including text and data mining) provided that all copyright notices and trade marks are retained.

\section{ORCID iDs}

Eric S Schwenk http://orcid.org/0000-0003-3464-4149

Rainish K Gupta http://orcid.org/0000-0003-3401-4737

Edward R Mariano http://orcid.org/0000-0003-2735-248X

\section{REFERENCES}

1 Gai N, Matava C. Twitter Hashtags for Anesthesiologists: building global communities. A A Pract 2019;12:59-62.

2 Jenkins WD, Bolinski R, Bresett J, et al. COVID-19 During the Opioid Epidemic - Exacerbation of Stigma and Vulnerabilities. J Rural Health 2020. doi:10.1111/ jrh.12442. [Epub ahead of print: 11 Apr 2020].

3 Hawksey M. \#TAGS History, \#TAGS, 2014. Available: https://tags. hawksey.info/about/ [Accessed 26 May 2020].

4 Utengen A. Healthcare Stakeholder segmentation, Symplur, 2020. Available: https:// help.symplur.com/en/articles/103684-healthcare-stakeholder-segmentation [Accessed 26 May 2020].

5 Schwenk ES, Jaremko KM, Gupta RK, et al. Upgrading a social media strategy to increase Twitter engagement during the spring annual meeting of the American Society of regional anesthesia and pain medicine. Reg Anesth Pain Med 2017;42:283-8.

6 McHugh ML. Interrater reliability: the kappa statistic. Biochem Med 2012;22:276-82. 